\title{
Existence and nonexistence results for a singular boundary value problem arising in the theory of epitaxial growth
}

\author{
Carlos Escudero* \\ Departamento de Matemáticas \& ICMAT (CSIC-UAM-UC3M-UCM), \\ Universidad Autónoma de Madrid, E-28049 Madrid, Spain \\ Robert Hakl ${ }^{\dagger}$ \\ Institute of Mathematics AS CR, Žižkova 22, 61662 Brno, Czech Republic \\ Ireneo Peral ${ }^{\ddagger}$ \\ Departamento de Matemáticas, \\ Universidad Autónoma de Madrid, E-28049 Madrid, Spain \\ Pedro J. Torres ${ }^{\S}$ \\ Departamento de Matemática Aplicada, \\ Universidad de Granada, E-18071 Granada, Spain.
}

\begin{abstract}
The existence of stationary radial solutions to a partial differential equation arising in the theory of epitaxial growth is studied. Our results depend on the size of a parameter that plays the role of the velocity at which mass is introduced into the system. For small values of this parameter we prove existence of solutions to this boundary value problem. For large values of the same parameter we prove nonexistence of solutions. We also provide rigorous bounds for the values of this parameter which separate existence from nonexistence. The proofs come as a combination of several differential inequalities and the method of upper and lower functions.
\end{abstract}

\footnotetext{
*Supported by projects MTM2010-18128, RYC-2011-09025 and SEV-2011-0087.

${ }^{\dagger}$ Supported by project RUO:67985840.

${ }^{\ddagger}$ Supported by project MTM2010-18128.

${ }^{\S}$ Supported by project MTM2011-23652.
} 


\section{Introduction}

Epitaxial growth is a technique used in the semiconductor industry for the growth of thin films [1]. It is employed for growing crystal structures by means of the deposition of a given material under high vacuum conditions. In epitaxial growth it is quite usual finding a mounded structure generated along the surface evolution rather than a flat surface [7]. A number of models has been considered in order to explain this phenomenology. These models have been usually introduced either as a discrete probabilistic system or as a differential equation [1]. In this work we are interested in this second type of modeling approach.

Here we will focus on the rigorous mathematical analysis of ordinary differential equations related to models which have been introduced in the context of epitaxial growth. The mathematical description of epitaxial growth uses the function

$$
\phi: \Omega \subset \mathbb{R}^{2} \times \mathbb{R}^{+} \rightarrow \mathbb{R}
$$

which describes the height of the growing interface in the spatial point $x \in \Omega \subset \mathbb{R}^{2}$ at time $\mathfrak{t} \in \mathbb{R}^{+}$. Although this theoretical framework can be extended to any spatial dimension $N$, we will concentrate here on the physical situation $N=2$. A basic modeling assumption is of course that $\phi$ is a well defined function, a fact that holds in a reasonably large number of cases [1]. The macroscopic description of the growing interface is given by a partial differential equation for $\phi$ which is usually postulated using phenomenological and symmetry arguments [1, 8]. There are many such equations in the theory of nonequilibrium surface growth. We will focus on the following one

$$
\partial_{\mathfrak{t}} \phi=\kappa_{1} \operatorname{det}\left(D^{2} \phi\right)-\kappa_{2} \Delta^{2} \phi+\xi(x, \mathfrak{t}) .
$$

This partial differential equation has been considered in the physical literature as a possible continuum description of epitaxial growth [3,4]. At the mathematical level we can consider it a parabolic problem whose evolution is dictated by a Monge-Ampère term stabilized by a fourth order viscosity.

In this work we are concerned with the stationary version of (1.2), which reads

$$
\left\{\begin{aligned}
\Delta^{2} \phi & =\operatorname{det}\left(D^{2} \phi\right)+\lambda F, \quad x \in \Omega \subset \mathbb{R}^{2}, \\
\text { boundary } & \text { conditions, }
\end{aligned}\right.
$$

after getting rid of the equation constant parameters by means of a trivial re-scaling of field and coordinates. Our last assumption is that the forcing term $F=F(x)$ is time independent. The constant $\lambda$ is a measure of the speed at which new particles are deposited, and for physical reasons we assume $\lambda \geq 0$ and $F(x) \geq 0$. We will devote our efforts to rigorously clarify the existence and nonexistence of solutions to this elliptic problem when set on a radially symmetric domain.

\section{Radial Solutions}

As already outlined in the previous section, our goal will be determining the existence of radially symmetric solutions of boundary value problem (1.3). We set the problem on 
the unit disk. In a previous work we have numerically found, for a constant forcing term $F(r) \equiv 1$, where $r$ is the radial coordinate, existence of two solutions for small values of $\lambda$ and nonexistence of any solution for sufficiently large values of $\lambda[5]$. In this paper we will still assume the forcing term is constant, and we will find rigorous bounds for the values of $\lambda$ separating existence from nonexistence. We note that we could also build the existence/nonexistence theory for arbitrary forcing terms $F(r)$ employing the same methods. However, in this case, of course, we would lose accuracy on the estimates of the bounds of $\lambda$.

In more precise terms, we look for solutions of the form $\phi=\tilde{\phi}(r)$ where $r=|x|$. By means of a direct substitution we find

$$
\frac{1}{r}\left\{r\left[\frac{1}{r}\left(r \tilde{\phi}^{\prime}\right)^{\prime}\right]^{\prime}\right\}^{\prime}=\frac{1}{r} \tilde{\phi}^{\prime} \tilde{\phi}^{\prime \prime}+\lambda F(r),
$$

where $^{\prime}=\frac{d}{d r}$. In the first case we consider homogeneous Dirichlet boundary conditions for problem (1.3). This translates to the conditions $\tilde{\phi}^{\prime}(0)=0, \tilde{\phi}(1)=0, \tilde{\phi}^{\prime}(1)=0$, and $\lim _{r \rightarrow 0} r \tilde{\phi}^{\prime \prime \prime}(r)=0$; the first one imposes the existence of an extremum at the origin and the second and third ones are the actual boundary conditions. The fourth boundary condition is technical and imposes higher regularity at the origin. If this condition were removed this would open the possibility of constructing functions $\tilde{\phi}(r)$ whose second derivative had a peak at the origin. This would in turn imply the presence of a measure at the origin when calculating the fourth derivative of such an $\tilde{\phi}(r)$, so this type of function cannot be considered as an acceptable solution of (2.1) whenever $F(r)$ is a function. Throughout this work we will assume $F \equiv 1$ as already mentioned.

Integrating once equation (2.1) against measure $r d r$ and using boundary condition $\lim _{r \rightarrow 0} r \tilde{\phi}^{\prime \prime \prime}(r)=0$ yields

$$
r\left[\frac{1}{r}\left(r \tilde{\phi}^{\prime}\right)^{\prime}\right]^{\prime}=\frac{1}{2}\left(\tilde{\phi}^{\prime}\right)^{2}+\frac{1}{2} \lambda r^{2} .
$$

By changing variables $w=r \tilde{\phi}^{\prime}$ we find the equation

$$
w^{\prime \prime}-\frac{1}{r} w^{\prime}=\frac{1}{2} \frac{w^{2}}{r^{2}}+\frac{1}{2} \lambda r^{2},
$$

subject to the conditions $w^{\prime}(0)=0$ and $w(1)=0$. This is the equation that has been numerically integrated in [5]; now we proceed to summarize these results. We observed that for $\lambda=0$ there are one trivial and one non-trivial solutions. For $0<\lambda<\lambda_{0}$ there are two non-trivial solutions which approach each other for increasing $\lambda$. For $\lambda>\lambda_{0}$ no more solutions were numerically found. The critical value of $\lambda$ was numerically estimated to be $\lambda_{0} \approx 169$. These results suggest no solutions exist for large enough $\lambda$.

In the second case we reconsider problem (1.3) but this time subject to homogeneous Navier boundary conditions. For the radial case we start as above, with equation (2.3), but now with the condition $w(1)=w^{\prime}(1)$ arbitrary instead of $w(1)=0$, what corresponds to homogeneous Navier boundary conditions. The results for this second case were qualitatively similar to those of the first case but quantitatively rather different [5]. 
In particular, we numerically observed that for $\lambda=0$ there are one trivial and one nontrivial solutions. For $0<\lambda<\lambda_{0}$ there are two non-trivial solutions which approach each other for increasing $\lambda$. For $\lambda>\lambda_{0}$ no more solutions were numerically found. The critical value of $\lambda$ was numerically estimated to be $\lambda_{0} \approx 11.34$.

\section{Statement of the Boundary Value Problem}

The rest of this work is devoted to rigorously justify the numerical facts summarized in the previous section. We will show how to proof existence of solutions for small $\lambda$ and nonexistence for large $\lambda$, as well as provide rigorous bounds for the values of this parameter which separate existence from nonexistence. Our first step will be recasting the differential equation under study into a form more suitable for its mathematical analysis.

Let us consider again equation (2.3), which we write now in the following form

$$
r^{2} w^{\prime \prime}-r w^{\prime}=\frac{\lambda}{2} r^{4}+\frac{w^{2}}{2} \quad \text { for } r \in(0,1]
$$

with $\lambda \geq 0$, together with the boundary conditions

$$
\begin{aligned}
& w^{\prime}(0)=0, \\
& w(1)=0,
\end{aligned}
$$

corresponding to Dirichlet boundary conditions, respectively the conditions (3.2) and

$$
w(1)=w^{\prime}(1)
$$

corresponding to Navier boundary conditions. The transformation

$$
t=\frac{r^{2}}{2}, \quad u(t)=w(r)
$$

leads to the equation

$$
u^{\prime \prime}=\frac{u^{2}}{8 t^{2}}+\frac{\lambda}{2} \quad \text { for } t \in(0,1 / 2]
$$

and the conditions

$$
\begin{gathered}
\lim _{t \rightarrow 0_{+}} \sqrt{t} u^{\prime}(t)=0, \\
u(1 / 2)=0,
\end{gathered}
$$

and

$$
u(1 / 2)=u^{\prime}(1 / 2) .
$$

Obviously, using the transformation (3.5) one can easily check that problems (3.1)-(3.3) (resp. (3.1), (3.2), (3.4)) and (3.6)-(3.8) (resp. (3.6), (3.7), (3.9)) are equivalent. By a solution to (3.6) we understand a function $u$ belonging to the space $C_{l o c}^{2}((0,1 / 2] ; \mathbb{R})$ of functions $u:(0,1 / 2] \rightarrow \mathbb{R}$ such that $u \in C^{2}([a, b] ; \mathbb{R})$ for every compact interval $[a, b] \subseteq(0,1 / 2]$, and satisfying (3.6) 


\section{Main Results}

We start with the case of the Dirichlet conditions.

Theorem 4.1. There exists a real number $\lambda_{0}>0$ such that problem (3.1)-(3.3) is solvable for every $\lambda \in\left[0, \lambda_{0}\right)$ and there is no solution to problem (3.1)-(3.3) if $\lambda>\lambda_{0}$. Furthermore, every solution $w$ to (3.1) - (3.3) satisfies

$$
w(r) \leq 0 \quad \text { for } r \in(0,1], \quad \lim _{r \rightarrow 0_{+}} w(r)=0 .
$$

The information contained in this Theorem is complementary to the existence and multiplicity results obtained in section 2 by means of variational arguments. On the other hand, the following Proposition gives us a localization of the critical value of $\lambda$ for problem (3.1)-(3.3).

Proposition 4.1. The number $\lambda_{0}$ from Theorem 4.1 admits the estimates

$$
144 \leq \lambda_{0} \leq 307
$$

The situation is analogous for the Navier conditions.

Theorem 4.2. There exists a real number $\lambda_{0}>0$ such that problem (3.1), (3.2), (3.4) is solvable for every $\lambda \in\left[0, \lambda_{0}\right)$ and there is no solution to problem (3.1), (3.2), (3.4) if $\lambda>\lambda_{0}$. Furthermore, every solution $w$ to (3.1), (3.2), (3.4) satisfies (4.1).

The following Proposition gives us a localization of the critical value of $\lambda$ for problem (3.1), (3.2), (3.4).

Proposition 4.2. The number $\lambda_{0}$ from Theorem 4.2 admits the estimates

$$
9 \leq \lambda_{0} \leq \frac{128}{11}=11 . \overline{63}
$$

By comparing these estimates with the numerical results, one observes that in both cases the rigorous bounds capture the order of magnitude of the critical value of the parameter. Estimates for the Navier problem are more accurate, and in this case the upper bound is rather precise.

The rest of this section is devoted to prove the latter results by means of a combination of techniques for differential inequalities and the method of upper and lower functions.

\section{$5 \quad$ Auxiliary Propositions}

In what follows, we establish some basic properties of a function $u \in C_{l o c}^{2}((0,1 / 2] ; \mathbb{R})$ satisfying the inequality

$$
u^{\prime \prime}(t) \geq \frac{u^{2}(t)}{8 t^{2}}+\frac{\lambda}{2} \quad \text { for } t \in(0,1 / 2] .
$$


Lemma 5.1. Let $u \in C_{\text {loc }}^{2}((0,1 / 2] ; \mathbb{R})$ satisfy (3.7) and (5.1). Then

$$
\lim _{t \rightarrow 0_{+}} u(t)=0 .
$$

Proof. From (5.1) it follows that $u^{\prime}$ is a non-decreasing function. Therefore, there exists $t_{0} \in(0,1 / 2]$ such that either

$$
u^{\prime}(t) \geq 0 \quad \text { for } t \in\left(0, t_{0}\right]
$$

or

$$
u^{\prime}(t)<0 \quad \text { for } t \in\left(0, t_{0}\right] .
$$

In both cases, the function $u$ is monotone on the interval $\left(0, t_{0}\right]$. Therefore, there exists a (finite or infinite) limit $u(0+)$. Assume that (5.2) does not hold. Then there exist $t_{1} \in(0,1 / 2]$ and $\delta>0$ such that

$$
u^{2}(t) \geq \delta \quad \text { for } t \in\left(0, t_{1}\right] .
$$

Thus the integration of (5.1) from $t$ to $t_{1}$ yields

$$
u^{\prime}\left(t_{1}\right)-u^{\prime}(t) \geq \frac{\delta}{8}\left(\frac{1}{t}-\frac{1}{t_{1}}\right) \quad \text { for } t \in\left(0, t_{1}\right] .
$$

Multiplying both sides of (5.3) by $\sqrt{t}$ and applying a limit as $t$ tends to zero we obtain

$$
-\lim _{t \rightarrow 0_{+}} \sqrt{t} u^{\prime}(t) \geq \frac{\delta}{8} \lim _{t \rightarrow 0_{+}} \frac{1}{\sqrt{t}}=+\infty,
$$

which contradicts (3.7).

Remark 5.1. Note that every function $u \in C_{l o c}^{2}((0,1 / 2] ; \mathbb{R})$ satisfying (3.7) and (5.1) satisfies also

$$
\lim _{t \rightarrow 0_{+}} \frac{u(t)}{\sqrt{t}}=0 .
$$

Indeed, the equality (5.4) follows from Lemma 5.1 and the l'Hospital rule.

On the other hand, if (5.4) holds then (5.2) is fulfilled.

Lemma 5.2. Let $u \in C_{\text {loc }}^{2}((0,1 / 2] ; \mathbb{R})$ satisfy (3.8), (5.2), and

$$
u^{\prime \prime}(t) \geq 0 \quad \text { for } t \in(0,1 / 2] .
$$

Then

$$
u(t) \leq 0 \quad \text { for } t \in(0,1 / 2] .
$$


Proof. Assume on the contrary that there exists $t_{0} \in(0,1 / 2]$ such that $u\left(t_{0}\right)>0$. According to (5.2) there exists $t_{1} \in\left(0, t_{0}\right)$ such that

$$
u\left(t_{1}\right)<u\left(t_{0}\right) .
$$

On the other hand, in view of (3.8) and (5.5) we have

$$
u(t) \leq \frac{u\left(t_{1}\right)(1 / 2-t)}{1 / 2-t_{1}} \quad \text { for } t \in\left[t_{1}, 1 / 2\right] .
$$

However, the latter inequality contradicts (5.7).

Lemma 5.3. Let $u \in C_{l o c}^{2}((0,1 / 2] ; \mathbb{R})$ satisfy (3.9), (5.2), and (5.5). Then (5.6) holds.

Proof. First we will show that

$$
u(1 / 2) \leq 0 .
$$

Assume on the contrary that (5.8) does not hold. Then, according to (5.2), there exists $t_{0} \in(0,1 / 2)$ such that

$$
u\left(t_{0}\right)<u(1 / 2)\left(1 / 2+t_{0}\right) .
$$

Obviously, (5.5) yields

$$
u^{\prime}(1 / 2) \geq \frac{u(1 / 2)-u\left(t_{0}\right)}{1 / 2-t_{0}},
$$

whence, in view of (3.9), we obtain

$$
u(1 / 2) \geq \frac{u(1 / 2)-u\left(t_{0}\right)}{1 / 2-t_{0}},
$$

However, the latter inequality contradicts (5.9). Therefore, the inequality (5.8) holds.

Finally, (3.9), (5.5), and (5.8) imply

$$
u^{\prime}(t) \leq 0 \quad \text { for } t \in(0,1 / 2],
$$

which together with (5.2) results in (5.6).

Lemma 5.4. Let $u \in C_{l o c}^{2}((0,1 / 2] ; \mathbb{R})$ satisfy (5.4)-(5.6). Then (3.7) is fulfilled.

Proof. In view of Remark 5.1 the equality (5.2) holds. Therefore we have

$$
u(t)=\int_{0}^{t} u^{\prime}(s) d s \quad \text { for } t \in(0,1 / 2] .
$$

According to (5.5), from (5.10) it follows that

$$
u(t) \leq t u^{\prime}(t) \quad \text { for } t \in(0,1 / 2] .
$$

Moreover, (5.2), (5.5), and (5.6) imply the existence of $t_{0} \in(0,1 / 2]$ such that $u^{\prime}(t) \leq 0$ for $t \in\left(0, t_{0}\right]$ which, together with (5.11), results in

$$
\frac{u(t)}{\sqrt{t}} \leq \sqrt{t} u^{\prime}(t) \leq 0 \quad \text { for } t \in\left(0, t_{0}\right] .
$$

Now we get (3.7) from (5.12) using (5.4). 
Lemma 5.5. The problems (3.6) -(3.8) (resp. (3.6), (3.7), (3.9)) and (3.6), (3.8), (5.4) (resp. (3.6), (3.9), (5.4)) are equivalent.

Proof. Let $u$ be a solution to (3.6)-(3.8) (resp. (3.6), (3.7), (3.9)). Then, according to Remark 5.1, $u$ is also a solution to (3.6), (3.8), (5.4) (resp. (3.6), (3.9), (5.4)).

On the other hand, let $u$ be a solution to (3.6), (3.8), (5.4) (resp. (3.6), (3.9), (5.4)). Then, in view of (3.6), Remark 5.1 and Lemma 5.2 (resp. Lemma 5.3), the inequalities (5.5) and (5.6) hold. Thus we get that (3.7) is fulfilled from Lemma 5.4.

Remark 5.2. It follows from Lemmas 3.1 3.3 that every solution $u$ to (3.6)-(3.8) (resp. (3.6), (3.7), (3.9) ) satisfies (5.2) and (5.6).

Lemma 5.6. Let $u \in C_{\text {loc }}^{2}((0,1 / 2] ; \mathbb{R})$ satisfy (5.4). Then for every $\mu \in[0,1)$ we have

$$
\lim _{t \rightarrow 0_{+}} t^{1-\mu} \int_{t}^{1 / 2} \frac{u^{2}(s)}{s^{2}} d s=0 .
$$

Proof. Put

$$
f(t)=\frac{u^{2}(t)}{t} \quad \text { for } t \in(0,1 / 2], \quad g(t)=\frac{1}{t^{\mu}} \quad \text { for } t \in(0,1 / 2] .
$$

Then, obviously, $g \in L([0,1 / 2] ; \mathbb{R})$ and in view of (5.4), $f \in L^{+\infty}([0,1 / 2] ; \mathbb{R})$. Consequently, $f g \in L([0,1 / 2] ; \mathbb{R})$. Therefore, for every $n \in \mathbb{N}$ there exists $t_{n} \in(0,1 / 2]$ such that

$$
\int_{0}^{t_{n}} \frac{u^{2}(s)}{s^{1+\mu}} d s \leq \frac{1}{n}
$$

On the other hand, for any $n \in \mathbb{N}$, we have

$$
\begin{aligned}
& 0 \leq t^{1-\mu} \int_{t}^{1 / 2} \frac{u^{2}(s)}{s^{2}} d s \leq t^{1-\mu} \int_{t_{n}}^{1 / 2} \frac{u^{2}(s)}{s^{2}} d s+t^{1-\mu} \int_{t}^{t_{n}} \frac{u^{2}(s)}{s^{2}} d s \leq \\
& \leq t^{1-\mu} \int_{t_{n}}^{1 / 2} \frac{u^{2}(s)}{s^{2}} d s+\int_{t}^{t_{n}} \frac{s^{1-\mu} u^{2}(s)}{s^{2}} d s \leq \\
& \leq t^{1-\mu} \int_{t_{n}}^{1 / 2} \frac{u^{2}(s)}{s^{2}} d s+\int_{t}^{t_{n}} \frac{u^{2}(s)}{s^{1+\mu}} d s \quad \text { for } t \in\left(0, t_{n}\right]
\end{aligned}
$$

and so, in view of (5.14),

$$
0 \leq \limsup _{t \rightarrow 0_{+}} t^{1-\mu} \int_{t}^{1 / 2} \frac{u^{2}(s)}{s^{2}} d s \leq \int_{0}^{t_{n}} \frac{u^{2}(s)}{s^{1+\mu}} d s \leq \frac{1}{n} \quad \text { for } n \in \mathbb{N} .
$$

Consequently, (5.13) holds. 
Lemma 5.7. Let $u \in C_{l o c}^{2}((0,1 / 2] ; \mathbb{R})$ satisfy (3.6) and (5.4). Then

$$
\begin{gathered}
u(t)=-\left[(1 / 2-t) \int_{0}^{t} \frac{u^{2}(s)}{4 s} d s+t \int_{t}^{1 / 2} \frac{u^{2}(s)}{4 s^{2}}(1 / 2-s) d s\right. \\
\left.+\frac{\lambda}{4} t(1 / 2-t)-2 t u(1 / 2)\right] \quad \text { for } t \in(0,1 / 2], \\
\lim _{t \rightarrow 0_{+}} \frac{u(t)}{t^{\mu}}=0 \quad \text { for } \mu \in[0,1),
\end{gathered}
$$

and there exists the finite limit

$$
\lim _{t \rightarrow 0_{+}} \frac{u(t)}{t}<+\infty
$$

Proof. For any $\tau \in(0,1 / 2)$ we have a representation

$$
\begin{gathered}
u(t)=-\frac{1}{1 / 2-\tau}\left[(1 / 2-t) \int_{\tau}^{t} \frac{u^{2}(s)}{8 s^{2}}(s-\tau) d s+(t-\tau) \int_{t}^{1 / 2} \frac{u^{2}(s)}{8 s^{2}}(1 / 2-s) d s\right. \\
\left.+\frac{\lambda}{4}(t-\tau)(1 / 2-t)(1 / 2-\tau)-u(\tau)(1 / 2-t)-u(1 / 2)(t-\tau)\right] \quad \text { for } t \in[\tau, 1 / 2] .
\end{gathered}
$$

According to (5.4) and Lemma 5.6 we have

$$
\lim _{\tau \rightarrow 0_{+}} \int_{\tau}^{t} \frac{u^{2}(s)}{s^{2}}(s-\tau) d s=\lim _{\tau \rightarrow 0_{+}} \int_{\tau}^{t} \frac{u^{2}(s)}{s} d s-\lim _{\tau \rightarrow 0_{+}} \tau \int_{\tau}^{t} \frac{u^{2}(s)}{s^{2}} d s=\int_{0}^{t} \frac{u^{2}(s)}{s} d s
$$

and, in view of Remark 5.1, (5.2) holds. Thus, on account of (5.20) and (5.2), from (5.19) it follows that (5.16) holds.

Now if we multiply both sides of (5.16) by $t^{-\mu}$ and apply a limit as $t$ tends to zero, with respect to Lemma 5.6 and (5.4), we obtain that (5.17) holds true.

Finally, put

$$
f(t)=\frac{u^{2}(t)}{t^{1+\mu}} \quad \text { for } t \in(0,1 / 2], \quad g(t)=\frac{1}{t^{1-\mu}} \quad \text { for } t \in(0,1 / 2] .
$$

Then, in view of (5.17), we have $f \in L^{+\infty}([0,1 / 2] ; \mathbb{R})$ and $g \in L([0,1 / 2] ; \mathbb{R})$ provided $\mu \in(0,1)$. Consequently, $f g \in L([0,1 / 2] ; \mathbb{R})$, i.e.,

$$
\int_{0}^{1 / 2} \frac{u^{2}(s)}{s^{2}} d s<+\infty
$$

Now from (5.16), in view of (5.4) and (5.21), we get

$$
\lim _{t \rightarrow 0_{+}} \frac{u(t)}{t}=\left|\int_{0}^{1 / 2} \frac{u^{2}(s)}{4 s^{2}}(1 / 2-s) d s+\frac{\lambda}{8}-2 u(1 / 2)\right|
$$

i.e., (5.18) holds. 


\section{$6 \quad$ Upper and Lower Functions}

First we will recall the notion of lower and upper functions to the general equation

$$
u^{\prime \prime}=h\left(t, u, u^{\prime}\right),
$$

where $h \in \operatorname{Car}\left([a, b] \times \mathbb{R}^{2} ; \mathbb{R}\right)$ is a Carathéodory function.

Definition 6.1. A continuous function $\gamma:[a, b] \rightarrow \mathbb{R}$ is said to be a lower (upper) function to (6.1) if $\gamma \in A C_{l o c}^{1}\left([a, b] \backslash\left\{t_{1}, \ldots, t_{n}\right\} ; \mathbb{R}\right)$, where $a<t_{1}<\cdots<t_{n}<b$, there exist finite limits $\gamma^{\prime}\left(t_{i}+\right), \gamma^{\prime}\left(t_{i}-\right)(i=1, \ldots, n)$,

$$
\gamma^{\prime}\left(t_{i}-\right)<\gamma^{\prime}\left(t_{i}+\right) \quad\left(\gamma^{\prime}\left(t_{i}-\right)>\gamma^{\prime}\left(t_{i}+\right)\right) \quad(i=1, \ldots, n),
$$

and the inequality

$$
\gamma^{\prime \prime}(t) \geq h\left(t, \gamma(t), \gamma^{\prime}(t)\right) \quad\left(\gamma^{\prime \prime}(t) \leq h\left(t, \gamma(t), \gamma^{\prime}(t)\right)\right) \quad \text { for a. e. } t \in[a, b]
$$

holds.

The following two lemmas deal with the existence of a solution to (6.1) satisfying the boundary conditions

$$
u(a)=c_{1}, \quad u(b)=c_{2},
$$

and

$$
u(a)=c_{1}, \quad u(b)=u^{\prime}(b) .
$$

The first one is a simple modification of Scorza Dragoni theorem and its proof can be found in [6] (see also the more recent monograph [2]).

Lemma 6.1. Let $\alpha$ and $\beta$ be, respectively, lower and upper functions to (6.1) such that

$$
\alpha(t) \leq \beta(t) \quad \text { for } t \in[a, b]
$$

and

$$
|h(t, x, y)| \leq q(t) \quad \text { for a. e. } t \in[a, b], \quad \alpha(t) \leq x \leq \beta(t), \quad y \in \mathbb{R},
$$

where $q \in L\left([a, b] ; \mathbb{R}_{+}\right)$. Then, for every $c_{1} \in[\alpha(a), \beta(a)]$ and $c_{2} \in[\alpha(b), \beta(b)]$, the problem (6.1), (6.2) has a solution $u \in A C^{1}([a, b] ; \mathbb{R})$ satisfying the condition

$$
\alpha(t) \leq u(t) \leq \beta(t) \quad \text { for } t \in[a, b] .
$$

Lemma 6.2. Let $\alpha$ and $\beta$ be, respectively, lower and upper functions to (6.1) satisfying (6.4) and

$$
\alpha(b) \geq \alpha^{\prime}(b), \quad \beta(b) \leq \beta^{\prime}(b) .
$$

Let, moreover, (6.5) is fulfilled where $q \in L\left([a, b] ; \mathbb{R}_{+}\right)$. Then, for every $c_{1} \in[\alpha(a), \beta(a)]$, the problem (6.1), (6.3) has a solution $u \in A C^{1}([a, b] ; \mathbb{R})$ satisfying (6.6). 
Proof. Let $c_{1} \in[\alpha(a), \beta(a)]$ be arbitrary but fixed. According to Lemma 6.1 there exists a solution $u_{1}$ to the equation (6.1) satisfying

$$
\begin{gathered}
u_{1}(a)=c_{1}, \quad u_{1}(b)=\alpha(b), \\
\alpha(t) \leq u_{1}(t) \leq \beta(t) \quad \text { for } t \in[a, b] .
\end{gathered}
$$

On account of (6.7)-( 6.9$)$ we have

$$
u_{1}(b) \geq u_{1}^{\prime}(b)
$$

Furthermore, $u_{1}$ can be considered as a lower function to (6.1) and so, according to Lemma 6.1, there exists a solution $v_{1}$ to the equation (6.1) satisfying

$$
\begin{gathered}
v_{1}(a)=c_{1}, \quad v_{1}(b)=\beta(b), \\
u_{1}(t) \leq v_{1}(t) \leq \beta(t) \quad \text { for } t \in[a, b] .
\end{gathered}
$$

On account of (6.7), (6.10), and (6.11) we have

$$
v_{1}(b) \leq v_{1}^{\prime}(b)
$$

Now we will construct a sequences of solutions to (6.1) $\left\{u_{n}\right\}_{n=1}^{+\infty}$ and $\left\{v_{n}\right\}_{n=1}^{+\infty}$ in the following way: Having defined solutions $u_{n}$ and $v_{n}$ for some $n \in \mathbb{N}$ with

$$
\begin{gathered}
u_{n}(a)=c_{1}, \quad v_{n}(a)=c_{1}, \quad u_{n}(b) \geq u_{n}^{\prime}(b), \quad v_{n}(b) \leq v_{n}^{\prime}(b), \\
\alpha(t) \leq u_{n}(t) \leq v_{n}(t) \leq \beta(t) \quad \text { for } t \in[a, b]
\end{gathered}
$$

we can consider them as a lower and an upper function, respectively, to (6.1). According to Lemma 6.1, there exists a solution $u$ to (6.1) satisfying

$$
\begin{gathered}
u(a)=c_{1}, \quad u(b)=\frac{u_{n}(b)+v_{n}(b)}{2}, \\
u_{n}(t) \leq u(t) \leq v_{n}(t) \quad \text { for } t \in[a, b] .
\end{gathered}
$$

Obviously, either

$$
u(b) \leq u^{\prime}(b)
$$

or

$$
u(b)>u^{\prime}(b)
$$

If (6.16) holds we put

$$
u_{n+1}(t)=u_{n}(t) \quad \text { for } t \in[a, b], \quad v_{n+1}(t)=u(t) \quad \text { for } t \in[a, b] .
$$

If (6.17) holds we put

$$
u_{n+1}(t)=u(t) \quad \text { for } t \in[a, b], \quad v_{n+1}(t)=v_{n}(t) \quad \text { for } t \in[a, b] .
$$


Consequently, in view of (6.12)-(6.19),$u_{n+1}$ and $v_{n+1}$ are solutions to (6.1) satisfying

$$
\begin{gathered}
u_{n+1}(a)=c_{1}, \quad v_{n+1}(a)=c_{1}, \quad u_{n+1}(b) \geq u_{n+1}^{\prime}(b), \quad v_{n+1}(b) \leq v_{n+1}^{\prime}(b), \\
\alpha(t) \leq u_{n}(t) \leq u_{n+1}(t) \leq v_{n+1}(t) \leq v_{n}(t) \leq \beta(t) \quad \text { for } t \in[a, b] .
\end{gathered}
$$

Obviously, in view of (6.14) and (6.18), resp. (6.19), and (6.20)

$$
\lim _{n \rightarrow+\infty} u_{n}(b)=\lim _{n \rightarrow+\infty} v_{n}(b) .
$$

Moreover, in view of (6.12), for any $n \in \mathbb{N}$, there exist $t_{n} \in[a, b]$ and $s_{n} \in[a, b]$ such that

$$
u_{n}^{\prime}\left(t_{n}\right)=\frac{u_{n}(b)-c_{1}}{b-a}, \quad v_{n}^{\prime}\left(s_{n}\right)=\frac{v_{n}(b)-c_{1}}{b-a} .
$$

Consequently, on account of (6.20), we have

$$
\left|u_{n}^{\prime}\left(t_{n}\right)\right| \leq M, \quad\left|v_{n}^{\prime}\left(s_{n}\right)\right| \leq M \quad \text { for } n \in \mathbb{N},
$$

where

$$
M=\frac{|\alpha(b)|+|\beta(b)|+\left|c_{1}\right|}{b-a} .
$$

Since $u_{n}$ and $v_{n}$ are solutions to (6.1), with respect to (6.5), (6.20), and (6.22) we obtain

$$
\begin{array}{ll}
\left|u_{n}^{\prime}(t)\right| \leq M+\int_{a}^{b} q(s) d s \quad \text { for } t \in[a, b], & n \in \mathbb{N}, \\
\left|v_{n}^{\prime}(t)\right| \leq M+\int_{a}^{b} q(s) d s \quad \text { for } t \in[a, b], \quad n \in \mathbb{N} .
\end{array}
$$

Thus, on account of (6.1), (6.20), (6.23), and (6.24), it follows that the sequences $\left\{u_{n}\right\}_{n=1}^{+\infty}$, $\left\{u_{n}^{\prime}\right\}_{n=1}^{+\infty}$ and $\left\{v_{n}\right\}_{n=1}^{+\infty},\left\{v_{n}^{\prime}\right\}_{n=1}^{+\infty}$ are uniformly bounded and equicontinuous. Therefore, without loss of generality we can assume that there exists functions $u_{0}, v_{0} \in C^{2}([a, b] ; \mathbb{R})$ such that

$$
u_{0}^{(j)}(t)=\lim _{n \rightarrow+\infty} u_{n}^{(j)}(t), \quad v_{0}^{(j)}(t)=\lim _{n \rightarrow+\infty} v_{n}^{(j)}(t) \quad \text { uniformly on }[a, b] \quad(j=0,1) .
$$

By a standard way it can be shown that $u_{0}, v_{0} \in A C^{1}([a, b] ; \mathbb{R})$, and they are also solutions to (6.1). Moreover, from (6.12), (6.20), and (6.21) we have

$$
\begin{gathered}
\alpha(t) \leq u_{0}(t) \leq v_{0}(t) \leq \beta(t) \quad \text { for } t \in[a, b], \\
u_{0}(a)=c_{1}, \quad v_{0}(a)=c_{1}, \quad u_{0}(b) \geq u_{0}^{\prime}(b), \quad v_{0}(b) \leq v_{0}^{\prime}(b), \\
u_{0}(b)=v_{0}(b) .
\end{gathered}
$$

On the other hand, from (6.25) and (6.27) it follows that

$$
u_{0}^{\prime}(b) \geq v_{0}^{\prime}(b)
$$

Now (6.25)-(6.28) imply that $u \stackrel{\text { def }}{=} u_{0}$ is a solution to (6.1), (6.3) satisfying (6.6). 
Lemma 6.3. Let there exists a function $\alpha \in C_{\text {loc }}^{2}((0,1 / 2] ; \mathbb{R})$ such that its restriction to any closed interval $[a, b] \subset(0,1 / 2]$ is a lower function to (3.6) on $[a, b]$. Let, moreover,

$$
\begin{gathered}
\alpha(t) \leq 0 \quad \text { for } t \in(0,1 / 2], \\
\lim _{t \rightarrow 0_{+}} \frac{|\alpha(t)|}{t}<+\infty .
\end{gathered}
$$

Then there exists a solution $u$ to (3.6), (3.8), (5.4) with

$$
\alpha(t) \leq u(t) \leq 0 \quad \text { for } t \in(0,1 / 2] .
$$

Proof. Note that from (6.30) it follows that

$$
\begin{gathered}
\lim _{t \rightarrow 0_{+}} \alpha(t)=0, \quad \lim _{t \rightarrow 0_{+}} \frac{\alpha(t)}{\sqrt{t}}=0, \\
\int_{0}^{1 / 2} \frac{\alpha^{2}(s)}{s^{2}} d s<+\infty .
\end{gathered}
$$

Further, from (6.32) it follows that

$$
\alpha^{*}=\sup \{|\alpha(t)|: t \in(0,1 / 2]\}<+\infty .
$$

Let $t_{n} \in(0,1 / 2)$ for $n \in \mathbb{N}$ be such that

$$
t_{n+1}<t_{n} \quad \text { for } n \in \mathbb{N}, \quad \lim _{n \rightarrow+\infty} t_{n}=0 .
$$

Obviously, for every $n \in \mathbb{N}, \beta \equiv 0$ is an upper function to (3.6) on the interval $\left[t_{n}, 1 / 2\right]$ satisfying

$$
\beta\left(t_{n}\right)=0, \quad \beta(1 / 2)=0 .
$$

Therefore, according to Lemma 6.1, in view of (6.29), for every $n \in \mathbb{N}$ there exists a solution $u_{n}$ to (3.6) on the interval $\left[t_{n}, 1 / 2\right]$ satisfying

$$
\begin{gathered}
u_{n}\left(t_{n}\right)=0, \quad u_{n}(1 / 2)=0, \\
\alpha(t) \leq u_{n}(t) \leq 0 \quad \text { for } t \in\left[t_{n}, 1 / 2\right] .
\end{gathered}
$$

Moreover, for every $n \in \mathbb{N}$ there exists $s_{n} \in\left(t_{n}, 1 / 2\right)$ such that

$$
u_{n}^{\prime}\left(s_{n}\right)=0 \text {. }
$$

Therefore, integrating (3.6) from $s_{n}$ to $t$, on account of (6.33), (6.37), and (6.38), we obtain

$$
\begin{array}{r}
\left|u_{n}^{\prime}(t)\right|=\left|\int_{s_{n}}^{t} \frac{u_{n}^{2}(s)}{8 s^{2}} d s+\frac{\lambda}{2}\left(t-s_{n}\right)\right| \leq \int_{0}^{1 / 2} \frac{\alpha^{2}(s)}{8 s^{2}} d s+\frac{\lambda}{4} \quad \text { for } t \in\left[t_{n}, 1 / 2\right], \\
n \in \mathbb{N} .
\end{array}
$$


Moreover, from (3.6) and (6.37) we get

$$
\left|u_{n}^{\prime \prime}(t)\right| \leq \frac{\alpha^{2}(t)}{8 t^{2}}+\frac{\lambda}{2} \quad \text { for } t \in\left[t_{n}, 1 / 2\right], \quad n \in \mathbb{N} .
$$

Thus, on account of (6.33)-(6.35), (6.37), (6.39), and (6.40), we have that the sequences $\left\{u_{n}\right\}_{n=1}^{+\infty},\left\{u_{n}^{\prime}\right\}_{n=1}^{+\infty}$ are uniformly bounded and equicontinuous on every compact subinterval of $(0,1 / 2]$. Therefore, according to Arzelà-Ascoli theorem, there exists $u_{0} \in$ $C_{\text {loc }}^{1}((0,1 / 2] ; \mathbb{R})$ such that

$$
\lim _{n \rightarrow+\infty} u_{n}^{(j)}(t)=u_{0}^{(j)}(t) \quad \text { uniformly on every compact interval of }(0,1 / 2] \quad(j=0,1) .
$$

Moreover, since $u_{n}$ are solutions to (3.6) $), u_{0} \in C_{l o c}^{2}((0,1 / 2] ; \mathbb{R})$ and it is also a solution to (3.6). Furthermore, from (6.32), (6.36), and (6.37), it follows that

$$
\alpha(t) \leq u_{0}(t) \leq 0 \quad \text { for } t \in(0,1 / 2], \quad u_{0}(1 / 2)=0, \quad \lim _{t \rightarrow 0_{+}} \frac{u_{0}(t)}{\sqrt{t}}=0 .
$$

Lemma 6.4. Let the assumptions of Lemma 6.3 be fulfilled. Let, moreover,

$$
\alpha(1 / 2) \geq \alpha^{\prime}(1 / 2)
$$

Then there exists a solution $u$ to (3.6), (3.9), (5.4) satisfying (6.31).

The proof of Lemma 6.4 is similar to that of Lemma 6.3, just Lemma 6.2 is used instead of Lemma 6.1 and the estimate of $u_{n}^{\prime}$ is produced using the fact that there exists $s_{n} \in\left(t_{n}, 1 / 2\right)$ such that

$$
\left|u_{n}^{\prime}\left(s_{n}\right)\right|=\frac{\left|u_{n}(1 / 2)\right|}{1 / 2-t_{n}} \leq \frac{|\alpha(1 / 2)|}{1 / 2-t_{1}} .
$$

\section{Lemmas on Estimation of $\lambda$}

Lemma 7.1. The set of numbers $\lambda \geq 0$, for which there exists a solution to (3.6) satisfying (5.4) and (5.6), is nonempty and bounded from above.

Proof. Obviously, for $\lambda=0$ there is a zero solution with the appropriate properties. Therefore the set is nonempty. If $\lambda=0$ is the only element of the set, then, clearly, the set is bounded from above. Let, therefore, $\lambda>0$ and let $u$ be a solution to (3.6) satisfying (5.4) and (5.6). Then, according to Remark 5.1 and Lemma 5.4, we have that (3.7) and (5.2) hold.

On the other hand, from (3.6) it follows that

$$
\left(t u^{\prime}(t)-u(t)\right)^{\prime}=\frac{u^{2}(t)}{8 t}+\frac{\lambda}{2} t \quad \text { for } t \in(0,1 / 2]
$$


Integration (7.1) from 0 to $t$, in view of (3.7), (5.2), and (5.4), yields

$$
t u^{\prime}(t)-u(t)=\int_{0}^{t} \frac{u^{2}(s)}{8 s} d s+\frac{\lambda}{4} t^{2} \quad \text { for } t \in(0,1 / 2]
$$

Put

$$
v(t)=-\frac{u(t)}{t} \quad \text { for } t \in(0,1 / 2]
$$

Then, on account of (5.6) and (17.2) we have

$$
\begin{gathered}
v^{\prime}(t)=-\frac{1}{8 t^{2}} \int_{0}^{t} v^{2}(s) s d s-\frac{\lambda}{4} \quad \text { for } t \in(0,1 / 2] \\
v(t) \geq 0 \quad \text { for } t \in(0,1 / 2] .
\end{gathered}
$$

Moreover, from (7.3) it follows that $v$ is a decreasing function and thus (7.3) and (7.4) result in

$$
v^{\prime}(t) \leq-\frac{v^{2}(t)+4 \lambda}{16} \quad \text { for } t \in(0,1 / 2]
$$

whence we get

$$
-\frac{v^{\prime}(t)}{v^{2}(t)+4 \lambda} \geq \frac{1}{16} \quad \text { for } t \in(0,1 / 2] .
$$

Now the integration of (7.5) from $t$ to $1 / 2$ results in

$$
\int_{v(1 / 2)}^{v(t)} \frac{d x}{x^{2}+4 \lambda}=-\int_{t}^{1 / 2} \frac{v^{\prime}(s) d s}{v^{2}(s)+4 \lambda} \geq \frac{1 / 2-t}{16} .
$$

Hence we get

$$
\frac{\pi}{4 \sqrt{\lambda}}=\int_{0}^{+\infty} \frac{d x}{x^{2}+4 \lambda} \geq \lim _{t \rightarrow 0_{+}} \int_{v(1 / 2)}^{v(t)} \frac{d x}{x^{2}+4 \lambda} \geq \frac{1}{32} .
$$

Consequently, (7.6) implies $\lambda \leq 64 \pi^{2}$.

Lemma 7.2. If the problem (3.6), (3.8), (5.4) (resp. (3.6), (3.9), (15.4)) is solvable for some $\lambda_{0} \geq 0$ then it is solvable also for every $\lambda \in\left[0, \lambda_{0}\right]$.

Proof. Let $u$ be a solution to the problem (3.6), (3.8), (5.4) (resp. (3.6), (3.9), (5.4)) with $\lambda=\lambda_{0}$. Put $\alpha(t)=u(t)$. Then according to Remark 5.1, Lemma 5.2 (resp. Lemma 5.3), and Lemma 5.7, the function $\alpha$ satisfies the assumptions of Lemma 6.3 (resp. Lemma 6.4), where the equation (3.6) is considered with $\lambda \in\left[0, \lambda_{0}\right]$. Consequently, according to Lemma 6.3 (resp. Lemma 6.4), the problem (3.6), (3.8), (15.4) (resp. (3.6), (3.9), (5.4)) is also solvable.

Lemma 7.3. Let

$$
\lambda \leq 144
$$

Then there exists $\alpha \in C_{\text {loc }}^{2}((0,1 / 2] ; \mathbb{R})$ satisfying the assumptions of Lemma 6.3 . 
Proof. Put

$$
\alpha(t)=-48 t(1-\sqrt{2 t}) \quad \text { for } t \in(0,1 / 2] .
$$

Obviously, (6.29) and (6.30) hold. We will show that

$$
\alpha^{\prime \prime}(t) \geq \frac{\alpha^{2}(t)}{8 t^{2}}+\frac{\lambda}{2} \quad \text { for } t \in(0,1 / 2] .
$$

In view of (17.8) we have

$$
\alpha^{\prime \prime}(t)-\frac{\alpha^{2}(t)}{8 t^{2}}-72=\frac{72}{\sqrt{2 t}}(1-\sqrt{2 t})(1-2 \sqrt{2 t})^{2} \geq 0 \quad \text { for } t \in(0,1 / 2]
$$

and thus, on account of (7.7), the inequality (7.9) is fulfilled.

Lemma 7.4. Let

$$
\lambda \leq 9 .
$$

Then there exists $\alpha \in C_{\text {loc }}^{2}((0,1 / 2] ; \mathbb{R})$ satisfying the assumptions of Lemma 6.4.

Proof. Put

$$
\alpha(t)=-6 t(2-\sqrt{2 t}) \quad \text { for } t \in(0,1 / 2] .
$$

Obviously, (6.29), (6.30), and (6.41) hold. We will show that (77.9) is valid. In view of (7.11) we have

$$
\alpha^{\prime \prime}(t)-\frac{\alpha^{2}(t)}{8 t^{2}}-\frac{9}{2}=\frac{9}{2 \sqrt{2 t}}(2-\sqrt{2 t})(1-\sqrt{2 t})^{2} \geq 0 \quad \text { for } t \in(0,1 / 2]
$$

and thus, on account of (7.10), the inequality (7.9) is fulfilled.

Lemma 7.5. Let there exist a function $v \in C_{\text {loc }}^{2}((0,1 / 2] ; \mathbb{R}) \cap L^{+\infty}([0,1 / 2] ; \mathbb{R})$ satisfying

$$
\begin{gathered}
v^{\prime}(t)=-\frac{1}{8 t^{2}} \int_{0}^{t} v^{2}(s) s d s-\frac{\lambda}{4} \quad \text { for } t \in(0,1 / 2], \\
v(t) \geq 0 \quad \text { for } t \in(0,1 / 2], \\
v(1 / 2)=0 .
\end{gathered}
$$

Then

$$
\lambda \leq 384
$$

and

$$
v(t) \geq 192\left(1-\sqrt{1-\frac{\lambda}{384}}\right)(1 / 2-t) \quad \text { for } t \in(0,1 / 2] .
$$


Proof. From (7.12) it follows that

$$
v^{\prime}(t) \leq 0 \quad \text { for } t \in(0,1 / 2] .
$$

Furthermore, $v^{\prime} \in C_{l o c}^{2}((0,1 / 2] ; \mathbb{R})$ and

$$
v^{\prime \prime}(t)=\frac{1}{4 t^{3}} \int_{0}^{t} v^{2}(s) s d s-\frac{v^{2}(t)}{8 t} \quad \text { for } t \in(0,1 / 2] .
$$

From (7.18), on account of (7.13) and (7.17) we obtain

$$
v^{\prime \prime}(t) \geq 0 \quad \text { for } t \in(0,1 / 2] .
$$

Therefore, (7.12) and (7.19) yield

$$
v^{\prime}(t) \leq v^{\prime}(1 / 2)=-\frac{1}{2} \int_{0}^{1 / 2} v^{2}(s) s d s-\frac{\lambda}{4} \leq-\frac{\lambda}{4} \quad \text { for } t \in(0,1 / 2] .
$$

Now the integration of (7.20) from $t$ to $1 / 2$, with respect to (7.14), results in

$$
v(t) \geq \frac{\lambda}{4}(1 / 2-t) \quad \text { for } t \in(0,1 / 2] .
$$

Put

$$
c_{1}=\frac{\lambda}{4}, \quad c_{n+1}=\frac{c_{n}^{2}}{384}+\frac{\lambda}{4} \quad \text { for } n \in \mathbb{N} .
$$

We will show that for every $n \in \mathbb{N}$ the inequality

$$
v(t) \geq c_{n}(1 / 2-t) \quad \text { for } t \in(0,1 / 2]
$$

holds. Obviously, (7.21) and (7.22) yield the validity of (17.23) for $n=1$. Assume therefore that (7.23) holds for some $n \in \mathbb{N}$. Then from (7.20) we obtain

$$
\begin{aligned}
v^{\prime}(t) \leq-\frac{1}{2} \int_{0}^{1 / 2} v^{2}(s) s d s-\frac{\lambda}{4} \leq-\left(\frac{c_{n}^{2}}{2} \int_{0}^{1 / 2}(1 / 2-s)^{2} s d s+\frac{\lambda}{4}\right) & =-c_{n+1} \\
& \text { for } t \in(0,1 / 2] .
\end{aligned}
$$

The integration of (17.24) from $t$ to $1 / 2$, with respect to (7.14), results in

$$
v(t) \geq c_{n+1}(1 / 2-t) \quad \text { for } t \in(0,1 / 2]
$$

Thus (7.23) holds for every $n \in \mathbb{N}$. Moreover,

$$
c_{2}=\frac{c_{1}^{2}}{384}+\frac{\lambda}{4} \geq \frac{\lambda}{4}=c_{1} \geq 0
$$


and, assuming $c_{n} \geq c_{n-1} \geq 0$ for some $n \in \mathbb{N}$, we get

$$
c_{n+1}=\frac{c_{n}^{2}}{384}+\frac{\lambda}{4} \geq \frac{c_{n-1}^{2}}{384}+\frac{\lambda}{4}=c_{n} .
$$

Thus $\left\{c_{n}\right\}_{n=1}^{+\infty}$ is a non-decreasing sequence of numbers which are, on account of (7.23), bounded from above. Therefore, there exists $c_{0} \in \mathbb{R}$ such that

$$
c_{0}=\lim _{n \rightarrow+\infty} c_{n}
$$

and from (7.22) and (7.23) we obtain

$$
\begin{gathered}
c_{0}=\frac{c_{0}^{2}}{384}+\frac{\lambda}{4} \\
v(t) \geq c_{0}(1 / 2-t) \quad \text { for } t \in(0,1 / 2] .
\end{gathered}
$$

Now (7.25) implies (7.15) and

$$
c_{0} \geq 192\left(1-\sqrt{1-\frac{\lambda}{384}}\right)
$$

and, consequently, from (7.26) we get (7.16).

Lemma 7.6. Let there exist a function $v \in C_{\text {loc }}^{2}((0,1 / 2] ; \mathbb{R}) \cap L^{+\infty}([0,1 / 2] ; \mathbb{R})$ satisfying (7.12), (7.13), and

$$
v(1 / 2)=-v^{\prime}(1 / 2)
$$

Then

$$
\lambda \leq \frac{128}{11} .
$$

Proof. From (7.12) it follows that (7.17) is fulfilled. Further, $v^{\prime} \in C_{l o c}^{2}((0,1 / 2] ; \mathbb{R})$ and (7.18) holds. From (7.18), on account of (7.13) and (7.17) we obtain (7.19). Therefore, (7.12) and (7.19) yield

$$
v^{\prime}(t) \leq v^{\prime}(1 / 2)=-c
$$

where

$$
c=\frac{1}{2} \int_{0}^{1 / 2} v^{2}(s) s d s+\frac{\lambda}{4}
$$

Now the integration of $(7.29)$ from $t$ to $1 / 2$, results in

$$
v(1 / 2)-v(t) \leq-c(1 / 2-t) \quad \text { for } t \in(0,1 / 2],
$$

whence, with respect to (7.27) and (7.29), we get

$$
v(t) \geq c(3 / 2-t) \quad \text { for } t \in(0,1 / 2] .
$$


Now using (7.31) in (7.30) we obtain

$$
c \geq \frac{c^{2}}{2} \int_{0}^{1 / 2}(3 / 2-s)^{2} s d s+\frac{\lambda}{4}
$$

i.e.,

$$
\frac{11}{128} c^{2}-c+\frac{\lambda}{4} \leq 0
$$

However, (7.32) implies (7.28).

Lemma 7.7. Let $u \in C_{\text {loc }}^{2}((0,1 / 2] ; \mathbb{R})$ satisfy (3.6), (3.8), and (5.4). Then

$$
\lambda<307 \text {. }
$$

Proof. Assume that there exists $u \in C_{l o c}^{2}((0,1 / 2] ; \mathbb{R})$ satisfying (3.6), (3.8), (5.4). According to Remark 5.1 and Lemma 5.2, $u$ satisfies (5.6). Moreover, according to Lemma 5.7, $u$ satisfies (5.16) and (5.18). Put

$$
v(t)=-\frac{u(t)}{t} \quad \text { for } t \in(0,1 / 2]
$$

Then, in view of (3.8), (5.6), (5.16), and (5.18) we have that $v$ satisfies all the assumptions of Lemma 7.5. Consequently, (77.15) and (7.16) hold.

On the other hand, (3.8), (5.16), and (7.33) yield

$$
\begin{aligned}
v(t)=\left[\frac{(1 / 2-t)}{4 t} \int_{0}^{t} v^{2}(s) s d s+\int_{t}^{1 / 2} \frac{v^{2}(s)}{4}(1 / 2-s) d s+\frac{\lambda}{4}(1 / 2-t)\right] & \text { for } t \in(0,1 / 2] .
\end{aligned}
$$

Obviously, (7.12) results in (7.17), and thus, in view of (7.13), we have

$$
\int_{0}^{t} v^{2}(s) s d s \geq v^{2}(t) \frac{t^{2}}{2} \quad \text { for } t \in(0,1 / 2] .
$$

Now using (7.16) and (77.35) in (7.34) we obtain

$$
v(t) \geq \frac{(1 / 2-t) t}{8} v^{2}(t)+\frac{c^{2}}{4} \int_{t}^{1 / 2}(1 / 2-s)^{3} d s+\frac{\lambda}{4}(1 / 2-t) \quad \text { for } t \in(0,1 / 2],
$$

where

$$
c=192\left(1-\sqrt{1-\frac{\lambda}{384}}\right) .
$$

The inequality (17.36) means that for every fixed $t \in(0,1 / 2]$, the value of the function $v$ at the point $t$ is a solution to the quadratic inequality

$$
\frac{(1 / 2-t) t}{8} x^{2}-x+\frac{(1 / 2-t)}{4}\left[\frac{c^{2}(1 / 2-t)^{3}}{4}+\lambda\right] \leq 0
$$


Thus (7.38) results in

$$
f(\lambda, t) \stackrel{\text { def }}{=} \frac{(1 / 2-t)^{2} t}{8}\left[\frac{c^{2}(1 / 2-t)^{3}}{4}+\lambda\right] \leq 1 \quad \text { for } t \in(0,1 / 2]
$$

with $c$ given by (7.37). However, it can be verified by a direct calculation that

$$
f(307,1 / 8)>1
$$

Therefore, if $\lambda=307$, there is no solution to (3.6), (3.8), (5.4), and, according to Lemma 7.2, there is no solution to (3.6), (3.8), (5.4) for $\lambda \geq 307$ either.

Lemma 7.8. Let $u \in C_{\text {loc }}^{2}((0,1 / 2] ; \mathbb{R})$ satisfy (3.6), (3.9), and (15.4). Then (7.28) is fulfilled.

Proof. Assume that there exists $u \in C_{l o c}^{2}((0,1 / 2] ; \mathbb{R})$ satisfying (3.6) $)$ (3.9), (5.4). According to Remark 5.1 and Lemma 5.3, $u$ satisfies (5.6). Moreover, according to Lemma 5.7, $u$ satisfies (5.16) and (5.18). Define $v$ by (7.33). Then, in view of (3.9), (15.6), (5.16), and (5.18) we have that $v$ satisfies all the assumptions of Lemma 7.6. Consequently, (7.28) holds.

\section{Proofs of the Main Results}

Theorem 4.1 (resp. 4.2) follows from Remark 5.1, Lemmas 5.2 (resp. 5.3), 5.5, 7.1, 7.2, and transformation (3.5). Proposition 4.1 follows from Lemmas 5.5, 6.3, 7.3, 77.7, and transformation (3.5). Proposition 4.2 follows from Lemmas 5.5, 6.4, 7.4, 7.8, and transformation (3.5) .

\section{References}

[1] A.-L. Barabási and H. E. Stanley, Fractal Concepts in Surface Growth (Cambridge University Press, Cambridge, 1995).

[2] C. De Coster and P. Habets, Two-point bondary value problems, lower and upper solutions, Mathematics in Science and Engineering Vol. 205 (Elsevier 2006).

[3] C. Escudero, Geometric principles of surface growth, Phys. Rev. Lett. 101 (2008) 196102.

[4] C. Escudero and E. Korutcheva, Origins of scaling relations in nonequilibrium growth, J. Phys. A: Math. Theor. 45 (2012) 125005.

[5] C. Escudero, R. Hakl, I. Peral, and P. J. Torres, preprint.

[6] I. T. Kiguradze and B. L. Shekhter, Singular boundary-value problems for secondorder ordinary differential equations, J. Sov. Math. 43 (1988), No. 2, 2340-2417. 
[7] G. Lengel, R. J. Phaneuf, E. D. Williams, S. Das Sarma, W. Beard, and F. G. Johnson, Nonuniversality in mound formation during semiconductor growth, Phys. Rev. B 60 (1999) R8469-R8472.

[8] M. Marsili, A. Maritan, F. Toigo, and J. R. Banavar, Stochastic growth equations and reparametrization invariance, Rev. Mod. Phys. 68 (1996) 963-983. 